\title{
An Analysis of Two Fluid Layers Enclosed Between Two Non-Porous Surfaces
}

\author{
ASAD SALEM \\ Weisberg Department of Mechanical Engineering \\ Marshall University \\ 1 John Marshall Drive., Huntington, WV 25755 \\ UNITED STATES OF AMERICA
}

\begin{abstract}
The stability of a two-phase interface is a crucial occurrence that involves the design of many engineering applications. It correlates the spatial and droplet size-distributions of many fluid spraying applications and has a great effect on the estimations of the critical heat flux of systems that involves phase change or evaporation. However, the existing hydrodynamic models are only able to predict the stability of a plane fluid sheet, surrounded by an infinite pool of liquid. The case of a thin sheet of liquid surrounding a vapor sheet and enclosed between two walls has not been studied yet. The present paper solves this problem using a linearized stability analysis. Velocity potentials satisfying these conditions are introduced and a complete analysis is presented.
\end{abstract}

Key-Words: Fluid Flow Stability, Nonporous Cavity, Sinusoidal Wave, Dilatational Wave.

Received: May 28, 2021. Revised: November 25, 2021. Accepted: December 19, 2021. Published: January 11, 2022.

\section{Introduction}

There are numerous theoretical and experimental studies of fluid flow instability over the past few decades since the work of Shea and Hagerty [1] because of its prevalence in fluid and heat transfer analysis and stability. The stability of fluid flow in between non-porous medium channel finds many important applications in geothermal and geophysical engineering such as underground disposal of nuclear wastes, spreading of chemical pollutants in water-saturated soil and many other applications. Shea and Hagerty studied the stability of a liquid sheet surrounded by air. They used a potential flow model for their analysis. They assumed a potential function for the different flow regimes and employed a sinusoidal waveform for the initial disturbance. Further, the wave formation at the film surface considerably improves mass and heat transfer rates and play a vital role in the process equipment, such as falling film in absorption columns condensers, and evaporators, [2,3]. The stability of fluid flow between tow parallel walls of unknown surface condition was studied by Chamkha [4]. The study analysed the flow characteristics and thermal and electrical properties of the fluid flow. It showed that if any velocity profile is unstable for a particular value of Reynold's number, it will be unstable at a lower value of the Reynold's number for the two-dimensional disturbances. The linear flow stability of a contaminated fluid with a monolayer flow down a slippery non-porous inclined channel was studied by Bhat and Samanta [5]. Bhat and Samanta used Orr-Sommerfeld equation for analysis with Chebyshev spectral collection method to obtain numerical solutions. Salem 6] analysed the flow of two plane nonDarcy fluid flow in narrow rectangular cavity using Keller-box solution.

The present work attempts to explore the stability of two-phase flow between layers non-porous surfaces. The stability of vapor layers contained by a liquid pool is an important phenomenon that affects the design of many engineering devices. This situation can arise in many practical applications, for example, the flow between parallel plates with internal heat generation such as steam generators, or if boiling occurs in the narrow gaps of heat exchangers. It is desirable to study the parameters influencing critical heat flux and the vapor removal mechanism from the heating surface. However, the existing hydrodynamic models are only able to predict the stability of a plane fluid sheet surrounded by an infinite pool of liquid. Many hydrodynamic models have been developed to explain this phenomenon. The 
criterion for stable film boiling on a horizontal surface facing upward can be developed from Taylor instability. The stability of an interface of waveform between two fluids of different densities depends on the balance of surface tension energy and the sum of the kinetic and potential energies of the wave. This process is called Taylor's instability wavelength. In this process, the length of the wave grows fast and thus predominates during the collapse of an infinite plane horizontal interface.

When two immiscible fluids flow relative to each other along with the interface of separation, there is a maximum relative velocity above which a small disturbance of the interface will amplify and grow and thereby distort the flow. This phenomenon is known as the Helmholtz instability.

This paper solves a similar problem that includes the addition of a solid boundary surrounding the outer fluid. The instabilities are observed at the interface of the fluid sheet as the vapor layer starts building at the surface. The objective of this work is to find an expression for the growth rate of these instabilities. Figures are plotted for the growth rate versus frequency for different sheet velocities. The solut ion used a linearized stability model where squares of the velocities and their products are ignored. Velocity potentials satisfying these conditions are introduced and a complete analysis is presented.

\section{Problem Formulation}

The idealized system to be treated is shown in Figure 1. The inner fluid will be considered the vapor phase and the other fluid will be the liquid phase (this can be reversed easily). The width of the inner fluid sheet is "2 a" and the plates are a distance " $b$ " from the centerline of the inner fluid. The density of the vapor phase is $\rho_{\mathrm{V}}$ and that for the liquid phase is $\rho_{L_{1}}$ and $\rho_{L_{2}}$. We assume the vapor phase to be moving vertically with velocity $V$ through the surrounding liquid phase $L_{1}$ and $L_{2}$, which moves with velocity $V^{\prime}$. Following Rayleigh (1954) the following assumptions are made:

1. The velocity of the sheet is great enough so that the effect of gravity will be ignored.

2. Both fluids are inviscid.

3. The surface tension between the vapor and liquid phase is $T$.

4. The surface at $y= \pm a$ (at the interface) is disturbed by a small disturbance of amplitude $H$ and frequency $\omega$ but out of arbitrary phase to each other.

5. Flow is in steady-state conditions
6. Nonlinear terms such as the squares and products of the variables are ignored through the derivation of equations (a linearized theory is used).

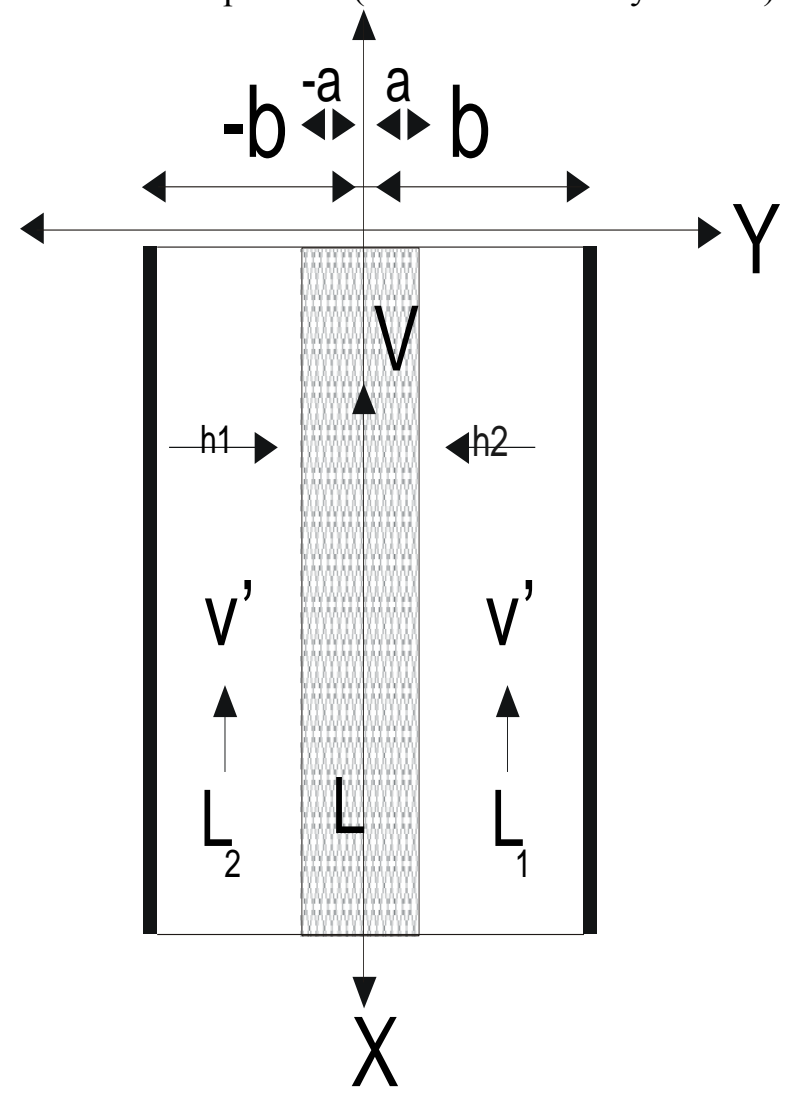

Fig. 1: Idealized fluid-sheet System

Based on the above assumptions, an incompressible inviscid flow between two parallel planes is assumed: $V=\nabla \Phi$ and $\nabla^{2} \Phi=0$, where $\Phi$ is the velocity potential function.

\section{Problem Solution}

Let us perturb the interface by a small wave of amplitude $H$. The equations of the disturbed surfaces (the interface) are assumed to be

and

$$
\square_{1}=H e^{i w t+i n x}, y=a
$$

$$
\square_{2}=H e^{i w t+i n x+i \varphi}, y=-a
$$

where phase angle $\varphi$ is introduced to treat both symmetrical and anti-symmetrical waves at the same time.

The boundary conditions are as follow:

1. The velocity of the fluid normal to the boundary is zero, i.e.

$$
\frac{D}{D t}\left[\square_{1}-y\right]=0, y=a
$$

and

$$
\frac{D}{D t}\left[\square_{2}-y\right]=0, y=-a
$$


2. the y-direction velocity at the wall is zero, i.e.

$$
\frac{\partial \Phi_{L_{1}}}{\partial y}=0, y=b
$$

and

$$
\frac{\partial \Phi_{L 2}}{\partial y}=0, y=-b
$$

the velocity potentials that satisfy the Laplace equation $\left(\nabla^{2} \Phi=0\right)$ as well as the boundary conditions as presented above, are given as follow, for the region $-a \leq y \leq a$, $\Phi_{V}=(A \cos \square(n y)+B \sin \square(n y)) e^{i w t+i n x}+V x$ (4a)

where

$$
A=\frac{i\left(\frac{w}{n}+V\right)\left(1-e^{i \varphi}\right)}{2 \sin \square(n a)} H
$$

and

$$
B=\frac{i\left(\frac{w}{n}+V\right)\left(1+e^{i \varphi}\right)}{2 \cos \square(n a)} H
$$

and the potential function for the surrounding fluid in the region $a \leq y \leq b$

$$
\Phi_{L_{1}}=C_{1} \frac{\cos \square(n(y-b))}{\sin \square(n(b-a))} e^{i w t+i n x}+V^{\prime} x
$$

where

$$
C_{1}=-i H\left(V^{\prime}+\frac{w}{n}\right)
$$

the velocity potential for the surrounding fluid in the region $-b \leq y \leq-a$

$$
\Phi_{L_{2}}=C_{2} \frac{\cos \square(n(y+b))}{\sin \square(n(b-a))} e^{i w t+i n x}+V^{\prime} x
$$

where

$$
C_{2}=-i H\left(V^{\prime}+\frac{w}{n}\right) e^{i \varphi}
$$

The velocity potential functions presented above satifies all the required conditions, the Laplace equation and the boundary conditions at the solid and the liquid-vapor interfaces.

\subsection{Pressure and Surface-Tension Forces}

The stability of the sheet depends on the growth rate of the disturbance at the liquid-vapor interface. The relationship between the surface tension and the pressure force acting at the surface of the sheet will determine the velocity of the vapor that will trigger the collapse of the sheet as in Helmholtz instability. The non-steady form of Bernoull's equation is given as

$$
\frac{V^{2}}{2}+\frac{P_{\text {ave }}}{\rho}=\frac{\partial \Phi}{\partial t}+\frac{q^{2}}{2}+\frac{P}{\rho}
$$

Then

$$
\Delta P=\rho\left(\frac{V^{2}}{2}-\frac{\partial \Phi}{\partial t}-\frac{q^{2}}{2}\right)
$$

Solving for the excess pressure by subsituting the velocity potentials into the equation above for the region $-a \leq y \leq a$ will yield

$$
\begin{gathered}
n \rho_{V}\left(\frac{w}{n}+V\right)^{2}\left(1-e^{i \varphi}\right) \cot \square n a+n \rho_{L}\left(\frac{w}{n}+\right. \\
\left.V^{\prime}\right)^{2}\left(1-e^{i \varphi}\right) \cot \square(n(b-a))=n^{2} T\left(1-e^{i \varphi}\right)
\end{gathered}
$$

$$
\left(1-e^{i \varphi}\right)=0
$$

or

and

$$
\begin{gathered}
n \rho_{V}\left(\frac{w}{n}+V\right)^{2} \cot \square n a+n \rho_{L}\left(\frac{w}{n}+\right. \\
\left.V^{\prime}\right)^{2} \cot \square(n(b-a))=n^{2} T \quad(8 \mathrm{~b})
\end{gathered}
$$

$$
\begin{gathered}
n \rho_{V}\left(\frac{w}{n}+V\right)^{2}\left(1+e^{i \varphi}\right) \tan \square n a+n \rho_{L}\left(\frac{w}{n}+\right. \\
\left.V^{\prime}\right)^{2}\left(1+e^{i \varphi}\right) \cot \square(n(b-a))=n^{2} T\left(1+e^{i \varphi}\right)
\end{gathered}
$$

$$
\left(1+e^{i \varphi}\right)=0
$$

or

$$
\begin{gathered}
n \rho_{V}\left(\frac{w}{n}+V\right)^{2} \tan \square n a+n \rho_{L}\left(\frac{w}{n}+\right. \\
\left.V^{\prime}\right)^{2} \cot \square(n(b-a))=n^{2} T(9 \mathrm{~b})
\end{gathered}
$$

Now, equation (9a) will yield $\varphi=\pi$ which corresponds to a dilatation wave solution while equation (8a) will yield $\varphi=0$ which corresponds to a sinusoidal wave solution.

\subsection{Analysis of the Sinusoidal and Di- latational Wave}

\subsubsection{Sinusoidal Wave}

For a sinuous wave $\varphi=0$, let $V^{\prime}=0$ and $\gamma=\frac{\rho_{V}}{\rho_{L}}$.

Equation (8) with rearrangement gives

$$
\begin{gathered}
\omega^{2}[\gamma \tan \square(n a)+\cot \square(n(b-a)]+ \\
2 \omega n V \tan \square(n a)+\gamma n^{2} V^{2} \tan \square(n a)-\frac{n^{3} T}{\rho_{L}}=0
\end{gathered}
$$

Solving equation (10) yields,

$$
\pm \frac{\omega=-n V \gamma \tan \square(n a)}{\gamma \tan \square(n a)+\cot \square(n(b-a))}
$$

For the particular case of interest, that of a fluid being sprayed into gas, the ratio $\gamma$ is quite small and can be neglected in comparison with $\tan \square(n a)$ over most of the unstable regions.

The unstable region consists of those waves which render the term under the radical negative. The sheet is unstable if

$$
n<\frac{\rho_{L} V^{2} \cot \square(n(b-a))}{T}
$$

From equation (11) the real circular frequency $\omega_{0}=n V$ and the upper bound of unstable frequencies is therefore

$$
f_{c}=\frac{\omega_{0}}{2 \pi}=\frac{n V}{2 \pi}=\frac{\rho_{L} V^{3} \cot \square(n(b-a))}{2 \pi T}
$$

For frequencies below $f_{c}$ the growth rate $\beta_{s}$ is 


$$
\begin{gathered}
\beta_{S}= \\
\frac{\sqrt{\gamma n^{2} V^{2} \tan \square(n a) \cot \square(n(b-a))-\frac{n^{3} T}{\rho_{L}}(\gamma \tan \square(n a)+\cot \square(n(b-a)))}}{\gamma \tan \square(n a)+\cot \square(n(b-a))}
\end{gathered}
$$

\subsubsection{Dilatational Wave}

For a dailational wave $\varphi=\pi, V^{\prime}=0$, and $\gamma=\frac{\rho_{V}}{\rho_{L}}$.

Now, frequency equation $(8 \mathrm{~b})$ is reduced to

$$
\begin{gathered}
\gamma \cot \square n a+\cot \square(n(b-a))+ \\
2 \omega n V \gamma \cot \square(n a) \gamma n^{2} V^{2} \cot \square(n a)-\frac{n^{3} T}{\rho_{L}}=0
\end{gathered}
$$

Assuming $\quad \gamma<<\cot \square(n a)$ over most of the unstable region

$$
\pm \frac{\omega=-\gamma n V \cot \square(n a)}{\gamma \operatorname{sot} \square(n a)+\cot \square(n(b-a))}
$$

This unstable region consists of those wave numbers $n$, which render the second term imaginary. The relationship is the same as in equation (12). As in the case of the sinuous wave, the upper bound of the unstable frequencies is given by equation (13). For the frequencies below $f_{c}$ the growth rate is $\beta_{d}$ is

$$
\beta_{d}=
$$$$
\frac{\sqrt{n^{2} V^{2} \gamma \cot \square(n a)+\cot \square(n(b-a))-\frac{n^{3} T}{\rho_{L}}(\gamma \cot \square(n a)+\cot \square(n(b-a)))}}{\gamma \cot \square(n a)+\cot \square(n(b-a))}
$$

\subsection{Representation of Equations}

\subsubsection{Logarithmic decrement $\Delta$ For Sinusoidal Wave}

The dominant wave in the unstable region has the form

$$
y=H e^{\beta t+i w t+i n x}
$$

During one complete cycle, $t$ increases by $\frac{2 \pi}{\omega}$, and $x$ increases by $\frac{2 \pi}{n}$. The ratio of the amplitude at the end of the cycle $y_{2}$, to the amplitude at the beginning of the cycle $y_{1}$ is

$$
\frac{y_{2}}{y_{1}}=e^{\frac{2 \pi \beta}{\omega}}=e^{\frac{\beta}{f}}(19)
$$

The logarithmic decrement $\Delta$ is defined as the natural logarthim of the ratio of the amplitude in successive cycles. Therefore

$$
\Delta=\ln \left(\frac{y_{2}}{y_{1}}\right)=\frac{\beta}{f}
$$

For the sinuous wave, using equations (13) and (14) and defining the dimensionless parameters as

$$
\begin{gathered}
\boldsymbol{\Omega}=\frac{f}{f_{c}} \\
W=\frac{V^{2} \rho_{L}(b-a)}{T}
\end{gathered}
$$

Equation (20) reduces to

$$
\Delta_{S}=\sqrt{\frac{\gamma \cot \square(W \Omega)-\gamma \Omega}{\tan \square(W \Omega)+\gamma \cot \square(W \Omega)}}
$$

whare $W$ is Weber's number.

\subsubsection{Logarithmic Decrement $\Delta_{d}$ For A Di- latational Wave}

Similarly, using equations (13), (19), (21), and (22), the logarithmic decrement for dalational wave reduces to

$$
\Delta_{D}=2 \pi \sqrt{\frac{\gamma \cot \square(W \Omega)-\gamma \Omega}{\cot \square(W \Omega)}}
$$

The growth rate $\beta$ could be plotted as a function of frequency $f$ for several different sheet velocities for both sinusoidal and dilatational waves, and $\Delta_{S}$ for various Weber numbers in the range of interest.

\section{Conclusion}

As a result of the theoretical work done in this paper, the following conclusions can be drawn. An expression for the growth rates as well as the logarithmic decrement for a sinusoidal and dilatational wave were determined, which helped to study the stability of plane fluid sheets with sidewalls. There are only two kinds of waves that are possible at any given frequency. Either the two surfaces of the sheet oscillate in phase to produce sinusoidal waves, or they oscillate out of phase as in a dilatational wave. The frequency, velocity, and wavelength are related in the same way for both types of waves. This relation is helpful in determining the velocity of the sheet. The equation is given by $f=\frac{V}{\lambda}$. It was, also, observed from the reduced functions, that the growth rate $\beta_{s}$ for a sinusoidal wave is always greater than that of a dilatational wave $\beta_{D}$ for the same given frequency. The equation is given by $\frac{\beta_{S}}{\beta_{D}}=\cot \square(n a)$.

\section{References:}

[1] "A Study of the Stability of Plane Fluid Sheets" by Hagerty, W.W. and Shea, J.F. 1955, Journal of Applied Mechanics, December 1955, pp 510-514. 
[2] "Gravity-driven flow of continuous thin liquid film on non-porous substrates with topography"". by P.H. Gaskell, P.K. Jimack, M. Seller, H. M. Thompson, and M.C. T. Wilson, J. Fluid Mech. (2004), Vol. 509, pp. 253-280.

[3] "Unsteady Flow Evolution in non-Porous Chamber with Surface Mass Injection, Part 1: Free Oscillation", by S. Apte, and V. Yang, AIAA Journal, Vol, 39, No. 8. August 2001.

[4] "Flow of Two-Immiscible Fluids in Porous and Nonporous Channels", by Ali J. Chamkha, Journal of Fluids Engineering Vol, 122, March 2000.

[5] "Linear stability of a contaminated fluid flow down a slippery inclined plane", by F. A. Bhat and A. Samanta, American Physical Society, 033108, 2018.

[6] " Analysis of Two Plane Fluid Layers in Narrow Rectangular Cavity', by Asad Salem, Proceedings of the 5th Inter. Conf. on Fluid Mechanics and Heat \&Mass Transfer, Lisbon Portugal, Oct. 30-Nov. 1, 2014

[7] " Micropolar fluid flow between a non-porous disk and a porous disk with slip: Keller-box solution", by A. Bhat and N. N. Katgi, Ain Shams Engineering Journal, 11 (2020), 149-159.
Creative Commons Attribution License 4.0 (Attribution 4.0 International, CC BY 4.0)

This article is published under the terms of the Creative Commons Attribution License 4.0

https://creativecommons.org/licenses/by/4.0/deed.en_US 